\title{
Realistic Area-Law Bound on Entanglement from Exponentially Decaying Correlations
}

\author{
Jaeyoon Cho \\ Asia Pacific Center for Theoretical Physics, Pohang 37673, Korea \\ and Department of Physics, POSTECH, Pohang 37673, Korea
}

\begin{abstract}
(Received 13 July 2017; revised manuscript received 12 June 2018; published 11 July 2018)
A remarkable feature of typical ground states of strongly correlated many-body systems is that the entanglement entropy is not an extensive quantity. In one dimension, there exists a proof that a finite correlation length sets a constant upper bound on the entanglement entropy, called the area law. However, the known bound exists only in a hypothetical limit, rendering its physical relevance highly questionable. In this paper, we give a simple proof of the area law for entanglement entropy in one dimension under the condition of exponentially decaying correlations. Our proof dramatically reduces the previously known bound on the entanglement entropy, bringing it into a realistic regime for the first time. The proof is composed of several simple and straightforward steps based on elementary quantum information tools. We discuss the underlying physical picture, based on a renormalization-like construction underpinning the proof, which transforms the entanglement entropy of a continuous region into a sum of mutual information in different length scales and the entanglement entropy at the boundary.
\end{abstract}

DOI: 10.1103/PhysRevX.8.031009

\section{INTRODUCTION}

Understanding the universal nature of strongly correlated many-body systems is one of the central topics in theoretical physics. Even though strongly correlated systems are generally intractable, it is possible to unfold the universal relationship between their characteristic attributes, providing a guiding principle for studying specific model Hamiltonians. For example, attributes commonly studied include the existence or absence of a spectral gap, a finite or diverging correlation length, and the behavior of entanglement entropies, which find intriguing mutual connections [1-13].

One of the prominent open problems in this context is whether the ground states of gapped Hamiltonians always obey the area law for entanglement entropy in any dimension, i.e., whether the entanglement between a subregion and its complement scales as the boundary size of the chosen region or can grow faster, e.g., as the volume of the region [1]. The underlying idea is that the existence of a gap significantly restricts the correlation that the ground state can accommodate. There is a well-established theorem, namely, the exponential-clustering theorem, which states that the existence of a spectral gap implies a finite correlation

Published by the American Physical Society under the terms of the Creative Commons Attribution 4.0 International license. Further distribution of this work must maintain attribution to the author(s) and the published article's title, journal citation, and DOI.
Subject Areas: Condensed Matter Physics,

Quantum Physics, Quantum Information length in the ground state [2-4]. Indeed, a seminal work by Hastings [5] as well as several ensuing works [6-8] have given proof of the area law in one-dimensional gapped systems, wherein the area law means a constant bound on the entanglement entropy. In higher-dimensional cases, however, only partial results are present $[9,10]$. Originally spawned by the Bekenstein-Hawking entropy $[14,15]$, the area law has gained a huge amount of interest over the last decade, thanks to its widespread relevance, e.g., to frameworks based on tensor network states $[16,17]$, topological entanglement entropies [18,19], the holographic formula based on the AdS/CFT correspondence [20], the Hamiltonian complexity theory [21], and so on.

After the proof for one-dimensional gapped systems, a naturally ensuing question became whether a finite correlation length alone could imply the area law. Albeit likely at first glance, serious doubt was cast upon its possibility because of unfavorable examples such as quantum datahiding states and quantum expander states, for which a small correlation and a large entanglement can coexist [11-13,22]. Amid such uncertainty, the recent proof that a finite correlation length indeed implies the area law in one dimension was a remarkable achievement $[12,13]$. However, the physical relevance of that proof is highly questionable because the upper bound of the entanglement entropy obtained is ridiculously huge, to such an extent that it is never reachable in any physically sensible situation (having a constant of about $10^{8}$ in the exponent, the bound easily surpasses the estimated number of atoms in the whole Universe) [13]. Consequently, we are still facing a 
situation that is quite unsatisfactory: Under the condition of a finite correlation length alone, does the upper bound of entanglement entropies exist only in such a hypothetical limit? Answering this question is important in truly confirming our picture of one-dimensional systems: In one dimension, a finite gap implies exponential decay of correlations, which in turn implies the area law. Here, the aforementioned unfavorable examples again seem to suggest that this picture might be misleading in reality.

In this paper, we give a proof of the one-dimensional entanglement area law from exponentially decaying correlations, which dramatically reduces the previously obtained bound and, for the first time, brings the bound into a realistic regime. In addition to the constants involved, our bound also improves the asymptotic dependence on the correlation length. With $\xi$ being the correlation length, we obtain a bound of $\sim(\log \xi) 2^{\text {(const }) \xi}$, while the previous proof gives $\sim \xi^{(\text {const }) \xi}[12,13]$. In fact, there are a number of strong cases for the exponential dependence on the correlation length being unavoidable (see Ref. [23] for the current state of understanding), which suggests that our bound leaves only little room for improvement. Interestingly, the dependence on $\xi$ is even more favorable than that of Hastings' original proof for gapped systems, which reads $\sim \xi(\log \xi) 2^{\text {(const) } \xi}$ [5], although this bound was significantly improved by a recent work (for gapped systems) [8].

Moreover, compared to the previous one, our proof is remarkably simpler and more straightforward. The proof directly addresses the internal structure of the states with exponentially decaying correlations using elementary quantum information tools. Such a direct nature allows us to envisage a clear and intuitive picture of the encountered situation. The central part of the proof shows that when the length scale is increased as $\ell_{0} \rightarrow x \ell_{0} \rightarrow x^{2} \ell_{0} \rightarrow$ $\cdots \rightarrow x^{n} \ell_{0}$ with $x>0$, the upper bound of the mutual information $I(A: C)$ in Fig. 1 initially increases indefinitely but saturates at some point and then decreases exponentially in $n$. Combined with a simple renormalization-like construction, this behavior of the mutual information accounts not only for the entanglement area law but also for why the area-law bound is exponentially large in the correlation length and how the common intuition (with a finite correlation length, the entanglement of a large region is determined by the correlations around the boundary) indeed makes sense.

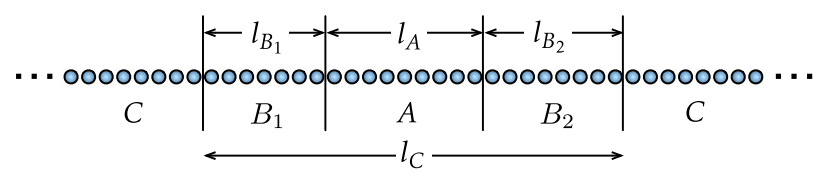

FIG. 1. The basic partitioning of the system for the proof. Here, $l_{B_{1}} \leq l_{B_{2}}$ as a convention. We let $l_{b}=l_{B_{1}}$ and $l_{B}=l_{B_{1}}+l_{B_{2}}$. Note that $l_{C}$ is defined to be $l_{A}+l_{B}$ because $S(C)=S(A B)$.
Thus, the present work makes our view on onedimensional systems quite solid and consistent. We hope that our proof offers a more direct and detailed insight into the situation and becomes an important step towards the understanding of the area law in higher dimensions.

\section{MAIN THEOREM}

We consider a one-dimensional chain of qubits, i.e., $s=1 / 2$ spins, in a pure state $\rho=|\Psi\rangle\langle\Psi|$. This setting covers the cases of higher-dimensional spins because one can then decompose each spin into a set of qubits and rescale the length. We make the following assumption:

Assumption. For arbitrary operators $X$ and $Y$ supported, respectively, on regions $R_{X}$ and $R_{Y}$, separated by a graph distance $l$, the following inequality holds:

$$
|\langle X \otimes Y\rangle-\langle X\rangle\langle Y\rangle| \leq\|X\|\|Y\| 2^{-l / \xi}
$$

where $\xi$ is the correlation length of the system, $\|\cdot\|$ denotes the operator norm, and $\langle\cdot\rangle=\operatorname{Tr}(\cdot \rho)$. Without loss of generality, we assume $\xi \geq 1$.

Under the above assumption, we prove the following theorem.

Theorem. For any real parameter $\alpha_{0} \in[2 / 3,1)$, the entanglement entropy $S$ of an arbitrary continuous region is bounded by

$$
S<\frac{\alpha_{0}}{1-\alpha_{0}}\left(\log \frac{\xi}{1-\alpha_{0}}+3\right) 4^{n_{0}}+12,
$$

where

$$
n_{0} \leq\left\lceil\frac{10 \xi}{\alpha_{0}}+\frac{1-\alpha_{0}}{\alpha_{0}} \frac{3}{\log \xi-\log \left(1-\alpha_{0}\right)+3}\right\rceil+2,
$$

with $\lceil x\rceil$ denoting the smallest integer larger than or equal to $x$.

For example, if $\alpha_{0}=10 / 11$ is taken, we obtain

$$
S<160(\log \xi+6.5) 4^{\lceil 11 \xi+0.05\rceil}+12 .
$$

We note that the theorem is obtained by a particular choice of the parameters used in the proof. Thus, the constants in the theorem do not represent the optimal ones. Having said that, it is nevertheless unlikely that the constants could be significantly reduced, unless the proof is enhanced by a completely new idea.

We also note that the main assumption (1) can be generalized to some extent. See Sec. VI for the details.

\section{OVERALL PICTURE}

Let us partition the chain into regions $A, B=B_{1}+B_{2}$, and $C$, as shown in Fig. 1. This partitioning is our basic setting throughout the proof. Our plan is to inspect the 


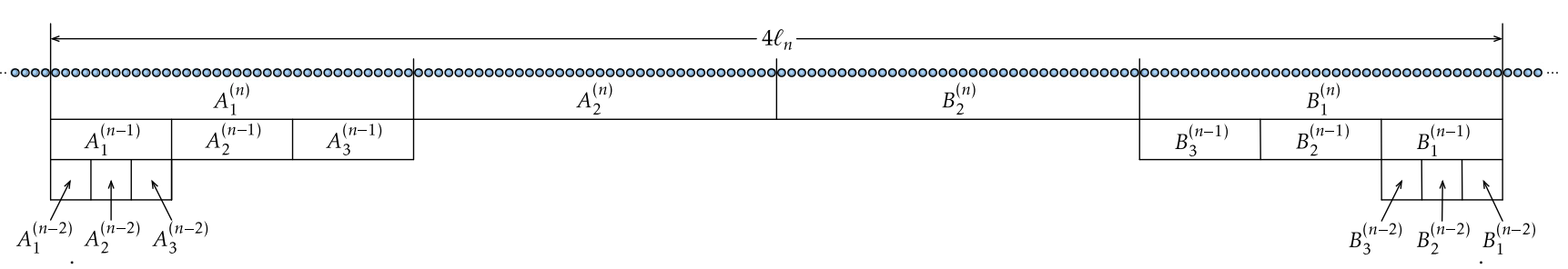

FIG. 2. Partitioning of the system for the proof of Lemma 7. The region of interest, which is of size $4 \ell_{n}$, is composed of four regions of size $\ell_{n}$. We recursively decompose the two end regions $A_{1}^{(i)}$ and $B_{1}^{(i)}$, respectively, into three regions of size $\ell_{i-1}$ following the rule $A_{1}^{(i)}=A_{1}^{(i-1)}+A_{2}^{(i-1)}+A_{3}^{(i-1)}$ and $B_{1}^{(i)}=B_{3}^{(i-1)}+B_{2}^{(i-1)}+B_{1}^{(i-1)}$. Each region denoted by superscript $(i)$ is of size $\ell_{i}=3^{i} \ell_{0}$.

entropic relations between the subregions while changing their sizes. The size of each region is denoted by $l$, with the corresponding subscript as in the caption of the figure. We denote the reduced density matrix by $\rho$, with the corresponding superscript, e.g., $\rho^{C}=\operatorname{Tr}_{A B} \rho$, and use a similar convention for other density matrices. The von Neumann entropy of a local region is denoted, e.g., by $S(C)=S\left(\rho^{C}\right)$, etc.

To begin, recall the definition of the mutual information:

$$
\begin{array}{r}
I(A: C)=S(A)+S(C)-S(A C) \geq 0, \\
I\left(B_{1}: B_{2}\right)=S\left(B_{1}\right)+S\left(B_{2}\right)-S(B) \geq 0 .
\end{array}
$$

One can rearrange the terms using $S(C)=S\left(B_{1} A B_{2}\right)$ and $S(A C)=S(B)$, ending up with a nice structure:

$$
S\left(B_{1} A B_{2}\right)=S\left(B_{1}\right)-S(A)+S\left(B_{2}\right)+f\left(B_{1}: A: B_{2}\right),
$$

where

$$
f\left(B_{1}: A: B_{2}\right) \equiv I(A: C)-I\left(B_{1}: B_{2}\right)
$$

is defined for later convenience.

The expression (6) can be utilized as follows. Consider the partitioning in Fig. 2. Let $\ell_{n}=3^{n} \ell_{0}$ with some small unit length $\ell_{0}$ and integer $n$. We are interested in the entanglement entropy of the large region $A_{1}^{(n)} A_{2}^{(n)} B_{2}^{(n)} B_{1}^{(n)}$ of length $4 \ell_{n}$. Equation (6) can be applied to this partitioning in two different ways:

$$
\begin{aligned}
S\left(A_{1}^{(n)} A_{2}^{(n)} B_{2}^{(n)} B_{1}^{(n)}\right)= & S\left(A_{1}^{(n)} A_{2}^{(n)}\right)-S\left(B_{2}^{(n)}\right)+S\left(B_{1}^{(n)}\right) \\
& +f\left(A_{1}^{(n)} A_{2}^{(n)}: B_{2}^{(n)}: B_{1}^{(n)}\right), \\
S\left(A_{1}^{(n)} A_{2}^{(n)} B_{2}^{(n)} B_{1}^{(n)}\right)= & S\left(A_{1}^{(n)}\right)-S\left(A_{2}^{(n)}\right)+S\left(B_{2}^{(n)} B_{1}^{(n)}\right) \\
& +f\left(A_{1}^{(n)}: A_{2}^{(n)}: B_{2}^{(n)} B_{1}^{(n)}\right) .
\end{aligned}
$$

Performing a similar task to the end regions, we obtain

$$
\begin{aligned}
S\left(A_{1}^{(i)} A_{2}^{(i)}\right)= & S\left(A_{1}^{(i-1)} A_{2}^{(i-1)}\right)-S\left(A_{3}^{(i-1)}\right)+S\left(A_{2}^{(i)}\right) \\
& +f\left(A_{1}^{(i-1)} A_{2}^{(i-1)}: A_{3}^{(i-1)}: A_{2}^{(i)}\right), \\
S\left(A_{1}^{(i)}\right)= & S\left(A_{1}^{(i-1)}\right)-S\left(A_{2}^{(i-1)}\right)+S\left(A_{3}^{(i-1)}\right) \\
& +f\left(A_{1}^{(i-1)}: A_{2}^{(i-1)}: A_{3}^{(i-1)}\right), \\
S\left(B_{1}^{(i)}\right)= & S\left(B_{3}^{(i-1)}\right)-S\left(B_{2}^{(i-1)}\right)+S\left(B_{1}^{(i-1)}\right) \\
& +f\left(B_{3}^{(i-1)}: B_{2}^{(i-1)}: B_{1}^{(i-1)}\right), \\
S\left(B_{2}^{(i)} B_{1}^{(i)}\right)= & S\left(B_{2}^{(i)}\right)-S\left(B_{3}^{(i-1)}\right)+S\left(B_{2}^{(i-1)} B_{1}^{(i-1)}\right) \\
& +f\left(B_{2}^{(i)}: B_{3}^{(i-1)}: B_{2}^{(i-1)} B_{1}^{(i-1)}\right) .
\end{aligned}
$$

Summing Eqs. (8) and (9) over $1 \leq i \leq n$, most of the $S(\cdot)$ terms are canceled out. The result is

$$
\begin{aligned}
S\left(A_{1}^{(n)}\right. & \left.A_{2}^{(n)} B_{2}^{(n)} B_{1}^{(n)}\right) \\
= & \frac{1}{2}\left\{S\left(A_{1}^{(0)} A_{2}^{(0)}\right)+S\left(A_{1}^{(0)}\right)-S\left(A_{2}^{(0)}\right)-S\left(B_{2}^{(0)}\right)\right. \\
& \left.+S\left(B_{1}^{(0)}\right)+S\left(B_{2}^{(0)} B_{1}^{(0)}\right)+f(\cdots)\right\} \\
= & S\left(A_{1}^{(0)}\right)+S\left(B_{1}^{(0)}\right)-\frac{1}{2}\left\{I\left(A_{1}^{(0)}: A_{2}^{(0)}\right)+I\left(B_{2}^{(0)}: B_{1}^{(0)}\right)\right\} \\
& +\frac{1}{2} f(\cdots),
\end{aligned}
$$

where $f(\cdots)$ is the abbreviation of the sum of all the mutual information terms in Eqs. (8) and (9) with different length scales. Note here that all the mutual information terms corresponding to $I(A: C)$ in Fig. 1 are added, whereas those corresponding to $I\left(B_{1}: B_{2}\right)$ are always subtracted.

The expression (10) reduces the problem into figuring out how the mutual information scales by varying the length scale. In the next section, we prove that if $\left\{l_{B_{1}}, l_{A}, l_{B_{2}}\right\}$ in Fig. 1 are all comparable to $x^{n} \ell_{0}$ with $x>0$, the behavior of the upper bound of $I(A: C)$ with respect to increasing $n$ is such that (i) it initially increases indefinitely for $n \lesssim n_{0}$, with $n_{0}$ being linearly large in the correlation length $\xi$ (transient behavior); however, (ii) it saturates around $n \simeq n_{0}$ (saturation), and then (iii) it decreases exponentially in $n$ for $n \gtrsim n_{0}$ (asymptotic behavior). A similar behavior can be proven for 
$I\left(B_{1}: B_{2}\right)$ by a slight modification of the proof, albeit not shown explicitly. Together with the expression (10), such behavior of the mutual information produces three straightforward implications.

First, the entanglement entropy of an arbitrarily large region of length $4 \ell_{n}$ is upper bounded by a constant as $f(\cdots)$ converges due to the asymptotic behavior of the mutual information. This leads to the entanglement area law as follows. For an arbitrary continuous region of length $l$, one can choose the partitioning in Fig. 1 such that $l_{A}=l$ and $l_{B_{1}}=l_{B_{2}}=4 \ell_{n}-l$ with sufficiently large $n$. Then, from the strong subadditivity $S(A) \leq S\left(B_{1} A\right)+S\left(A B_{2}\right)-S(C)$, we find that the entanglement entropy of an arbitrary region is upper bounded by twice the maximum entanglement entropy of a region of length $4 \ell_{n}$. This bound is the inequality (2) in the main theorem.

Second, the upper bound of the entanglement entropy is mostly determined by the transient and saturation behavior. As $n_{0}$ is linear in $\xi$, the area-law bound is exponentially large in the correlation length.

Third, Eq. (10) is valid for any choice of $\ell_{0}$. Our plan is to take the size of $A_{1}^{(0)}$ and $B_{1}^{(0)}$ to be the saturation length scale, which will be denoted by $l_{0}$, instead of a small unit length $\ell_{0}$. Then, the entanglement entropy is bounded by the entanglement entropies of the two boundary regions $A_{1}^{(0)}$ and $B_{1}^{(0)}$, where the remaining mutual information terms in $f(\cdots)$ are treated as a finite correction to the bound. The point to be addressed here is that if we change $l_{0}$ to $3^{m} l_{0}$ with positive integer $m$, then, while the size of the boundary region is increased, the sum of the remaining mutual information terms decays exponentially in $m$ due to the aforementioned asymptotic behavior. The physical meaning of this is that if the correlation length is finite, the entanglement entropy of a large region is determined by the correlations around the boundary, which is consistent with our common intuition. A caveat is that, as mentioned above, the boundary region responsible for the entanglement entropy is exponentially large in the correlation length, in general.

\section{EXISTENCE OF AN AREA-LAW BOUND}

The state of the system can be written in a Schmidtdecomposed form as

$|\Psi\rangle=\sum_{i=1}^{M} \sqrt{p_{i}}|i\rangle^{A} \otimes\left|\phi_{i}\right\rangle^{B C}, \quad p_{1} \geq p_{2} \geq \cdots \geq p_{M}$,

where the Schmidt coefficients $p_{i}$ are sorted in descending order and $M \leq 2^{l_{A}}$ is the Schmidt number. If a local projection

$$
P_{m n} \equiv \sum_{i=m}^{n}|i\rangle^{A}\langle i|
$$

on region $A$ is applied, the resulting normalized state of region $C$ is given by

$$
\rho_{m n}^{C} \equiv \sum_{i=m}^{n} \frac{p_{i}}{Q_{m n}} \phi_{i i}^{C}
$$

where we define

$$
\begin{gathered}
Q_{m n} \equiv\left\langle P_{m n}\right\rangle=\sum_{i=m}^{n} p_{i}, \\
\phi_{i j}^{C} \equiv \operatorname{Tr}_{B}\left|\phi_{i}\right\rangle^{B C}\left\langle\phi_{j}\right| .
\end{gathered}
$$

The exponential decay of correlations implies that the larger $Q_{m n}$ and $l_{b}$ are, the closer $\rho_{m n}^{C}$ is to the original state $\rho^{C}$ of region $C$ :

\section{Lemma 1.}

$$
\mathcal{D}\left(\rho^{C}, \rho_{m n}^{C}\right) \leq \frac{2^{-l_{b} / \xi}}{Q_{m n}}
$$

where

$$
\mathcal{D}(\rho, \sigma) \equiv \frac{1}{2}\|\rho-\sigma\|_{1}=\max _{0 \leq \Lambda \leq I} \operatorname{Tr}\{\Lambda(\rho-\sigma)\}
$$

denotes the trace distance between two states $\rho$ and $\sigma$.

Proof.-For any operator $\Lambda^{C}$ supported on region $C$,

$$
\begin{aligned}
\left|\operatorname{Tr}\left\{\Lambda^{C}\left(\rho^{C}-\rho_{m n}^{C}\right)\right\}\right| & =\left|\left\langle\left(I-\frac{P_{m n}}{Q_{m n}}\right) \otimes \Lambda^{C}\right\rangle\right| \\
& \leq\left\|I-\frac{P_{m n}}{Q_{m n}}\right\|\left\|\Lambda^{C}\right\| 2^{-l_{b} / \xi},
\end{aligned}
$$

where we use the assumption (1) and $\left\langle I-P_{m n} / Q_{m n}\right\rangle=0$. By choosing $0 \leq \Lambda^{C} \leq I$ that maximizes the trace, we obtain the lemma.

An important step is to define $q(\alpha) \in\{0,1, \ldots, M\}$, with a control parameter $\alpha \in(0,1)$ to be chosen later in such a way that the number of $p_{i}$ 's with $p_{i} \geq 2^{-\alpha l_{b} / \xi}$ is $q(\alpha)$. As $p_{i}$ 's are sorted in descending order, $q(\alpha)$ plays the role of a cutoff index in that $p_{q(\alpha)} \geq 2^{-\alpha l_{b} / \xi}$ and $p_{q(\alpha)+1}<2^{-\alpha l_{b} / \xi}$ unless $q(\alpha)=0$ or $M$. For $i \leq q(\alpha)$, each $\phi_{i i}^{C}$ alone is a good approximation to $\rho^{C}$ as $\mathcal{D}\left(\rho^{C}, \rho_{i i}^{C}\right) \leq 2^{-(1-\alpha) l_{b} / \xi}$ from Lemma 1. For $i>q(\alpha)$, on the other hand, individual $\phi_{i i}^{C}$, s are basically uncertain and should be considered arbitrary apart from the constraint in Lemma 1. This uncertain portion amounts to

$$
Q(\alpha) \equiv \sum_{p_{i}<2^{-\alpha l_{b} / \xi}} p_{i}
$$

For $q(\alpha)<M, Q(\alpha)=Q_{q(\alpha)+1, M}$, and if $q(\alpha)=M$, $Q(\alpha)=0$. Note that $Q(\alpha)$ is a function of region $A$ and $l_{b}$ in Fig. 1. For brevity, we simply denote it $Q(\alpha)$, the meaning of which will be clear from the context. We later see that when $\left\{l_{B_{1}}, l_{A}, l_{B_{2}}\right\}$ are all comparable, $Q(\alpha)$ 
asymptotically vanishes for a large length scale, which is an important part of the proof.

The Fannes' inequality states that for states $\rho$ and $\sigma$ acting on a Hilbert space of dimension $d$, if $\mathcal{D}(\rho, \sigma)=\epsilon \in[0,1]$, $|S(\rho)-S(\sigma)| \leq \epsilon \log d-\epsilon \log \epsilon-(1-\epsilon) \log (1-\epsilon)$ [24]. If $\epsilon \in[0,1 / 2]$, we can use a modified form

$$
|S(\rho)-S(\sigma)| \leq \epsilon(\log d-2 \log \epsilon)
$$

as $\epsilon \log \epsilon-(1-\epsilon) \log (1-\epsilon) \leq-2 \epsilon \log \epsilon$. From this modified Fannes' inequality, Lemma 1 directly leads to the following lemma.

Lemma 2. If $Q_{m n} \geq 2^{-\alpha l_{b} / \xi}$ and $(1-\alpha) l_{b} \geq \xi$, then

$$
\left|S(C)-S\left(\rho_{m n}^{C}\right)\right| \leq \epsilon\left(l_{B}+\log M, l_{b}, \alpha\right),
$$

where

$$
\epsilon\left(L, l_{b}, \alpha\right) \equiv\left\{L+2(1-\alpha) l_{b}\right\} 2^{-(1-\alpha) l_{b} / \xi} .
$$

Proof.-For $\quad Q_{m n} \geq 2^{-\alpha l_{b} / \xi}, \quad \mathcal{D}\left(\rho^{C}, \rho_{m n}^{C}\right) \leq 2^{-(1-\alpha) l_{b} / \xi}$ from Lemma 1. As the Hilbert-space dimension for region $C$ is upper bounded by $M 2^{l_{B}}$ from the expression (11), the Fannes' inequality (19) leads to the lemma.

Note that $\log M \leq l_{A}$. If $\left\{l_{B_{1}}, l_{A}, l_{B_{2}}\right\}$ are all comparable to each other, the bound in Lemma 2 decreases exponentially in $l_{b}$.

Let us define another important quantity, namely, $S(l) \in[0, l]$, which denotes the maximum entropy of a continuous region of length $l$. Furthermore, let us define the maximum entropy per site with a slight modification:

$$
\bar{s}(l) \equiv \frac{S(l)+\epsilon_{h}}{l},
$$

where

$$
\epsilon_{h} \equiv \max _{p \in[0,1]}(-p \log p)<0.531
$$

The additional constant is added merely for simplicity of the ensuing formulas. Note that from the subadditivity of entropy,

$$
\bar{s}(n l) \leq \bar{s}(l) \text { for any positive integer } n .
$$

It turns out that $Q(\alpha)$ is related to $\bar{s}(l)$ from the concavity of the entropy for region $A$.

\section{Lemma 3.}

$$
Q(\alpha) \leq \frac{\xi}{\alpha l_{b}}\left\{S(A)+\epsilon_{h}\right\} \leq \frac{\xi l_{A}}{\alpha l_{b}} \bar{s}\left(l_{A}\right) .
$$

Proof.-If $q(\alpha)=M$, the lemma is satisfied as $Q(\alpha)=0$. Suppose $q(\alpha)<M$. Given various possible cases of $\left\{p_{i}\right\}$, $S\left(\rho_{m M}^{A}\right)=-\sum_{i=m}^{M}\left(p_{i} / Q_{m M}\right) \log \left(p_{i} / Q_{m M}\right) \quad$ is minimal when $p_{i}=p_{m}$ for $m \leq i<M$ and $p_{M} \leq p_{m}$. Thus,

$$
\begin{aligned}
S\left(\rho_{m M}^{A}\right) & \geq \frac{Q_{m M}-p_{M}}{p_{m}}\left(-\frac{p_{m}}{Q_{m M}} \log \frac{p_{m}}{Q_{m M}}\right)-\frac{p_{M}}{Q_{m M}} \log \frac{p_{M}}{Q_{m M}} \\
& =-\frac{Q_{m M}-p_{M}}{Q_{m M}} \log p_{m}-\frac{p_{M}}{Q_{m M}} \log p_{M}+\log Q_{m M} \\
& \geq-\log p_{m}+\log Q_{m M} .
\end{aligned}
$$

From this and the concavity of entropy,

$$
\begin{aligned}
S(A) & \geq Q(\alpha) S\left(\rho_{q(\alpha)+1, M}^{A}\right) \\
& \geq Q(\alpha)\left(\frac{\alpha l_{b}}{\xi}+\log Q(\alpha)\right) \geq Q(\alpha) \frac{\alpha l_{b}}{\xi}-\epsilon_{h},
\end{aligned}
$$

which implies the lemma.

In addition, the concavity of the entropy for region $B$ results in the following lemma.

Lemma 4. If $(1-\alpha) l_{b} \geq \xi$, then

$$
S(C) \leq \frac{1}{1-Q(\alpha)} S(B)+\epsilon\left(l_{C}, l_{b}, \alpha\right) .
$$

Proof.-If $q(\alpha)=0$, the lemma is satisfied as $Q(\alpha)=1$. Suppose $q(\alpha)>0$. From the concavity of entropy,

$$
\begin{gathered}
S(B) \geq\{1-Q(\alpha)\} S\left(\rho_{1 q(\alpha)}^{B}\right), \\
S\left(\rho_{1 q(\alpha)}^{B}\right) \geq \sum_{i=1}^{q(\alpha)} \frac{p_{i}}{1-Q(\alpha)} S\left(\phi_{i i}^{B}\right) .
\end{gathered}
$$

The second inequality implies that there exists a certain $i_{0} \in\{1,2, \ldots, q(\alpha)\}$ such that $S\left(\phi_{i_{0} i_{0}}^{B}\right) \leq S\left(\rho_{1 q(\alpha)}^{B}\right)$. The lemma then follows because $S\left(\phi_{i_{0} i_{0}}^{B}\right)=S\left(\phi_{i_{0} i_{0}}^{C}\right)$ and $S(C) \leq$ $S\left(\phi_{i_{0} i_{0}}^{C}\right)+\epsilon\left(l_{C}, l_{b}, \alpha\right)$ from Lemma 2.

Suppose $l_{B_{1}}: l_{A}: l_{B_{2}}=1:(x-2): 1$ for some integer $x \geq 3$ and $l_{b}=l_{0}$; hence, $l_{C}=x l_{0}$. Note $S(B) \leq 2 S\left(l_{0}\right)$ from the subadditivity of entropy. If $l_{0}$ is sufficiently large so that $\epsilon\left(l_{C}, l_{b}, \alpha\right) \leq \epsilon_{h}$, Lemma 4 implies

$$
\bar{s}\left(x l_{0}\right) \leq \frac{1}{1-Q(\alpha)} \frac{2}{x} \bar{s}\left(l_{0}\right) .
$$

On the other hand, as $\bar{s}\left(l_{A}\right)=\bar{s}\left((x-2) l_{0}\right) \leq \bar{s}\left(l_{0}\right)$ from the subadditivity (24), $Q(\alpha)$ is also bounded by $\bar{s}\left(l_{0}\right)$ from Lemma 3. The inequality (29) thus becomes

$$
\bar{s}\left(x l_{0}\right) \leq \frac{1}{1-\{(x-2) \xi / \alpha\} \bar{s}\left(l_{0}\right)} \frac{2}{x} \bar{s}\left(l_{0}\right) \equiv \gamma\left(x, l_{0}\right) \bar{s}\left(l_{0}\right),
$$

where we define a function $\gamma(x, l)$ accordingly. Consequently, if there exists $l_{0}$ with sufficiently small $\bar{s}\left(l_{0}\right)$ so that $\gamma\left(x, l_{0}\right)<1$, then 


$$
\bar{s}\left(x^{n} l_{0}\right) \leq\left\{\prod_{i=0}^{n-1} \gamma\left(x, x^{i} l_{0}\right)\right\} \bar{s}\left(l_{0}\right)<\gamma_{0}^{n} \bar{s}\left(l_{0}\right)
$$

where $\gamma_{0} \equiv \gamma\left(x, l_{0}\right)$. Note that $\lim _{n \rightarrow \infty} \gamma\left(x, x^{n} l_{0}\right)=2 / x$ if $\gamma_{0}<1$.

The inequality (31) and Lemma 3 indicate that both $\bar{s}\left(x^{n} l_{0}\right)$ and $Q(\alpha)$ asymptotically decay exponentially in $n$. It turns out later that this is related to the asymptotic behavior of the mutual information mentioned in the previous section. Before proceeding, however, we need to make sure that there indeed exists such an $l_{0}$ that makes $\bar{s}\left(l_{0}\right)$ sufficiently small. Only then is the above argument valid. The inequality (29) is insufficient here as $Q(\alpha)$ may be arbitrarily close to 1 in the first place.

In order to obtain a complementary inequality, we revisit the subadditivity of entropy, which can be derived from the expression (11):

$$
\begin{aligned}
S(A B) & =S(C)=S\left(\sum_{i=1}^{M} p_{i} \phi_{i i}^{C}\right) \leq H\left(\left\{p_{i}\right\}\right)+\sum_{i=1}^{M} p_{i} S\left(\phi_{i i}^{C}\right) \\
& =H\left(\left\{p_{i}\right\}\right)+\sum_{i=1}^{M} p_{i} S\left(\phi_{i i}^{B}\right) \leq S(A)+S(B),
\end{aligned}
$$

where

$$
H\left(\left\{p_{i}\right\}\right) \equiv-\sum_{i} p_{i} \log p_{i}
$$

denotes the Shannon entropy. The second line is a general inequality [24], the third line uses $S\left(\phi_{i i}^{C}\right)=S\left(\phi_{i i}^{B}\right)$, and the last line uses the concavity of entropy. The following lemma is obtained by incorporating Lemma 2 into the inequality (32).

Lemma 5. If $Q(\alpha) \geq 2^{-\alpha l_{b} / \xi}$ and $(1-\alpha) l_{b} \geq \xi$, then

$S(C) \leq S(A)+S(B)-Q(\alpha) \frac{\alpha l_{b}}{\xi}+2 \epsilon_{h}+\epsilon\left(l_{C}, l_{b}, \alpha\right)$.

Proof.-Let us slightly modify the inequality (32). In other words, let us group $M p_{i}$ 's into $K \leq M$ sets as $\left\{R_{1}, R_{2}, \ldots, R_{K}\right\}$ with $R_{m}=Q_{i_{m}, i_{m+1}-1}$, where $i_{1}=1<i_{2}<\cdots$ $<i_{K+1}=M+1$. We then have

$$
\begin{aligned}
H\left(\left\{p_{i}\right\}\right) & =\sum_{m=1}^{K} R_{m} \sum_{i=i_{m}}^{i_{m+1}-1} \frac{p_{i}}{R_{m}}\left(-\log \frac{p_{i}}{R_{m}}-\log R_{m}\right) \\
& =-\sum_{m=1}^{K} R_{m} \sum_{i=i_{m}}^{i_{m+1}-1} \frac{p_{i}}{R_{m}} \log \frac{p_{i}}{R_{m}}+H\left(\left\{R_{m}\right\}\right)
\end{aligned}
$$

and

$$
\sum_{i=1}^{M} p_{i} S\left(\phi_{i i}^{C}\right)=\sum_{m=1}^{K} R_{m} \sum_{i=i_{m}}^{i_{m+1}-1} \frac{p_{i}}{R_{m}} S\left(\phi_{i i}^{C}\right) .
$$

But, again from the inequality

$$
\begin{aligned}
S\left(\rho_{i_{m}, i_{m+1}-1}^{C}\right) & =S\left(\sum_{i=i_{m}}^{i_{m+1}-1} \frac{p_{i}}{R_{m}} \phi_{i i}^{C}\right) \\
& \leq-\sum_{i=i_{m}}^{i_{m+1}-1} \frac{p_{i}}{R_{m}} \log \frac{p_{i}}{R_{m}}+\sum_{i=i_{m}}^{i_{m+1}-1} \frac{p_{i}}{R_{m}} S\left(\phi_{i i}^{C}\right),
\end{aligned}
$$

the inequality (32), along with Eqs. (35) and (36), becomes

$$
\sum_{m=1}^{K} R_{m} S\left(\rho_{i_{m}, i_{m+1}-1}^{C}\right) \leq S(A)+S(B)-H\left(\left\{R_{m}\right\}\right) .
$$

Let us now actually group the indices so that

$$
\begin{cases}i_{m}=i & \text { for } 1 \leq m \leq q(\alpha), \\ 2^{-\alpha l_{b} / \xi} \leq R_{m} \leq 2 \cdot 2^{-\alpha l_{b} / \xi} & \text { for } m>q(\alpha),\end{cases}
$$

which is always possible as $p_{i}<2^{-\alpha l_{b} / \xi}$ for $i>q(\alpha)$ and $Q(\alpha) \geq 2^{-\alpha l_{b} / \xi}$. Note $R_{m} \geq 2^{-\alpha l_{b} / \xi}$ for all $m$. Then, from Lemma $2, S(C) \leq S\left(\rho_{i_{m}, i_{m+1}-1}^{C}\right)+\epsilon\left(l_{C}, l_{b}, \alpha\right)$ for all $m$, and hence the inequality (38) becomes

$$
S(C) \leq S(A)+S(B)-H\left(\left\{R_{m}\right\}\right)+\epsilon\left(l_{C}, l_{b}, \alpha\right) .
$$

To obtain the lower bound of $H\left(\left\{R_{m}\right\}\right)$, we follow the same logic as in the proof of Lemma 3 and use the concavity of entropy to find

$$
\begin{aligned}
H\left(\left\{R_{m}\right\}\right) & \geq Q(\alpha)\left\{-\log \left(2 \cdot 2^{-\alpha l_{b} / \xi}\right)+\log Q(\alpha)\right\} \\
& \geq Q(\alpha) \frac{\alpha l_{b}}{\xi}+\min _{x \in[0,1]}(-x+x \log x) .
\end{aligned}
$$

As $\min _{x}(-x+x \log x)=\min _{x}\{-2 x+2 x \log (2 x)\}=-2 \epsilon_{h}$,

$$
H\left(\left\{R_{m}\right\}\right) \geq Q(\alpha) \frac{\alpha l_{b}}{\xi}-2 \epsilon_{h},
$$

which implies the lemma.

Suppose $l_{B_{1}}: l_{A}: l_{B_{2}}=1:(x-2): 1$ and $l_{b}=\ell_{0}$. If $x \geq 4$ and $\ell_{0}$ is sufficiently large so that $\epsilon\left(l_{C}, l_{b}, \alpha\right) \leq \epsilon_{h}$, Lemma 5 implies

$$
\bar{s}\left(x \ell_{0}\right) \leq \bar{s}\left(\ell_{0}\right)-Q(\alpha) \frac{\alpha}{x \xi},
$$

where we have used the subadditivity (24). It can be seen that the inequalities (29) and (42) can play complementary roles. Note that for any $Q_{c} \in(0,1)$, if $Q(\alpha) \leq Q_{c}, \bar{s}(x l) \leq$ $\left\{[2 \bar{s}(l)] /\left[x\left(1-Q_{c}\right)\right]\right\}$ from the inequality (29), and if 
$Q(\alpha) \geq Q_{c}, \bar{s}(x l) \leq \bar{s}(l)-Q_{c}[\alpha /(x \xi)]$ from the inequality (42). We thus find

$$
\bar{s}(x l) \leq \max \left\{\frac{2 \bar{s}(l)}{x\left(1-Q_{c}\right)}, \bar{s}(l)-Q_{c} \frac{\alpha}{x \xi}\right\} .
$$

For example, take $x=4$ and $Q_{c}=2 / 5$. Then, $\bar{s}(x l) \leq$ $\bar{s}(l)-\alpha / 10 \xi$ if $\bar{s}(l) \geq 3 \alpha / 5 \xi$ because, in this case, the second term on the right-hand side is larger than or equal to the first term. Consequently, if we assume $\bar{s}\left(x^{r} \ell_{0}\right) \geq 3 \alpha / 5 \xi$ for all positive integers $r, 0 \leq \bar{s}\left(x^{r} \ell_{0}\right) \leq \bar{s}\left(\ell_{0}\right)-r \alpha / 10 \xi$ leads to a contradiction, which means there exists $r \lesssim 10 \xi / \alpha$ such that $\bar{s}\left(x^{r} \ell_{0}\right)<3 \alpha / 5 \xi$. Once we reach this point, the inequality (43) implies $\bar{s}\left(x^{r+1} \ell_{0}\right)<(5 / 6) \bar{s}\left(x^{r} \ell_{0}\right)$ because now the first term on the right-hand side is larger than the second term. In the same manner, for positive integer $m$, $\bar{s}\left(x^{r+m} \ell_{0}\right)<(5 / 6)^{m}(3 \alpha / 5 \xi)$, which decreases exponentially in $m$ (in fact, the decrement is much faster because $Q_{c}$ can be adjusted optimally at each step). We thus come to a conclusion that for arbitrarily small $\epsilon>0$, there exists $l_{0}=x^{n_{0}} \ell_{0}$ such that $\bar{s}\left(l_{0}\right)<\epsilon$, with $n_{0}$ being linearly large in $\xi$ and $\log (1 / \epsilon)$; hence, the inequality (31) is indeed valid. The detailed calculation is presented in the next section.

The next step is to see how mutual information $I(A: C)$ scales by increasing the length scale as $l_{0} \rightarrow x l_{0} \rightarrow$ $x^{2} l_{0} \rightarrow \cdots$. As the mutual information quantifies the amount of information shared between two regions, the exponentially decaying correlation is expected to imply a decay of $I(A: C)$ with increasing length scale. The explicit bound of $I(A: C)$ can be obtained by separating the state $\rho^{A C}=\sum_{i, j=1}^{M} \sqrt{p_{i} p_{j}}|i\rangle^{A}\langle j| \otimes \phi_{i j}^{C}$ into three parts as

$$
\rho^{A C}=\left[\sum_{i, j=1}^{q(\alpha)}+\sum_{i, j=q(\alpha)+1}^{M}+\sum_{\text {the rest }}\right] \sqrt{p_{i} p_{j}}|i\rangle^{A}\langle j| \otimes \phi_{i j}^{C}
$$

and inspecting how each part contributes to $S(A C)$. From a rather straightforward analysis, we end up with the following lemma.

Lemma 6. For $\alpha \in(0,1 / 2)$, if $(1-2 \alpha) l_{b} \geq \xi$, then

$$
\begin{aligned}
I(A: C) \leq & 2 Q(\alpha) S(C)+2 H(\{Q(\alpha), 1-Q(\alpha)\}) \\
& +2 \epsilon\left(l_{C}, l_{b}, \alpha\right)+\epsilon\left(l_{B}+l_{b}, l_{b}, 2 \alpha\right) .
\end{aligned}
$$

If $Q(\alpha)<1 / 2$,

$$
\begin{aligned}
I(A: C) \leq & 2 Q(\alpha) S(C)-4 Q(\alpha) \log Q(\alpha) \\
& +2 \epsilon\left(l_{C}, l_{b}, \alpha\right)+\epsilon\left(l_{B}+l_{b}, l_{b}, 2 \alpha\right) .
\end{aligned}
$$

The detailed proof is given in the Appendix.

Suppose $\left\{l_{B_{1}}, l_{A}, l_{B_{2}}\right\}$ are small-integer multiples of $x^{n} l_{0}$. Then, $Q(\alpha) S(C) \leq \mathcal{O}\left[\bar{s}\left(l_{A}\right) \bar{s}\left(l_{C}\right) l_{C}\right] \leq \mathcal{O}\left[\bar{s}\left(x^{n} l_{0}\right)^{2} l_{C}\right] \leq$ $\mathcal{O}\left[\left(\gamma_{0}^{2} x\right)^{n}\right]$ from Lemma 3 , the subadditivity (24), and the inequality (31), where $\mathcal{O}[\cdot]$ omits the constant prefactor. Similarly, $\quad-Q(\alpha) \log Q(\alpha) \leq \mathcal{O}\left[n \gamma_{0}^{n}\right]+\mathcal{O}\left[\gamma_{0}^{n}\right]$ from Lemma 3. Consequently, if $\gamma_{0}<1 / \sqrt{x}$, the right-hand side of the inequality (44) is exponentially small in $n$. This is possible with $x>4$ and sufficiently small $\bar{s}\left(l_{0}\right)$, as can be seen from the inequality (30).

We are now fully ready for the final stage of the proof. Let us define

$$
\eta\left(l_{1}, l_{2}, l_{3}\right) \equiv \max _{l_{B_{1}}=l_{1}, l_{A}=l_{2}, l_{B_{2}}=l_{3}} I(A: C)
$$

Then, from the expression (10), we get the following lemma.

Lemma 7. For $l_{n}=3^{n} l_{0}$,

$$
S\left(4 l_{n}\right) \leq 2 S\left(l_{0}\right)+\eta\left(l_{n}, l_{n}, 2 l_{n}\right)+\sum_{0 \leq i<n} \lambda_{i},
$$

where $\lambda_{i} \equiv \eta\left(2 l_{i}, l_{i}, 3 l_{i}\right)+\eta\left(l_{i}, l_{i}, l_{i}\right)$.

This lemma states that $S\left(4 l_{n}\right)$ is upper bounded by a constant $2 S\left(l_{0}\right)$ independent of $n$, up to the correction terms. The remaining task is to ensure that the correction terms are indeed upper bounded by a constant. This is easily done in view of our analysis above. We have shown above that

$$
\begin{aligned}
I(A: C) \leq & \mathcal{O}\left[\left(\gamma_{0}^{2} x\right)^{n}\right]+\mathcal{O}\left[n \gamma_{0}^{n}\right]+\mathcal{O}\left[\gamma_{0}^{n}\right] \\
& +\mathcal{O}\left[l_{b} 2^{-(1-\alpha) l_{b} / \xi}\right]+\mathcal{O}\left[l_{b} 2^{-(1-2 \alpha) l_{b} / \xi}\right]
\end{aligned}
$$

when $\left\{l_{B_{1}}, l_{A}, l_{B_{2}}\right\}$ are small-integer multiples of $x^{n} l_{0}$ with $x>4$, where it is understood that $\bar{s}\left(l_{0}\right)$ can be made arbitrarily small so that $\gamma_{0}^{2} x<1$. This directly implies that $\lambda_{2 m}$ decays exponentially in $m$ as $l_{2 m}=9^{m} l_{0}$ (i.e., $x=9$ ); hence, $\sum_{m=0}^{\infty} \lambda_{2 m}$ is a finite constant. Likewise, $\lambda_{2 m+1}$ also decays exponentially in $m$; hence, $\sum_{m=0}^{\infty} \lambda_{2 m+1}$ is a finite constant. As a result, $\sum_{i=0}^{\infty} \lambda_{i}$ is upper bounded by a constant, as we anticipated.

The analytical bound can be obtained by summing the right-hand side of the inequality (48) over $n$ and restoring the omitted constant prefactors according to Lemma 7. For example, from the inequality (45) and Lemma 3,

$$
\begin{aligned}
\eta\left(2 l_{2 i}, l_{2 i}, 3 l_{2 i}\right)< & 2 \frac{\xi}{2 \alpha} \bar{s}\left(l_{2 i}\right) \bar{s}\left(6 l_{2 i}\right) 6 l_{2 i} \\
& -4 \frac{\xi}{2 \alpha} \bar{s}\left(l_{2 i}\right) \log \left\{\frac{\xi}{2 \alpha} \bar{s}\left(l_{2 i}\right)\right\}
\end{aligned}
$$

where we use the fact that $\epsilon(\cdots)$ terms are much smaller than the $2[\xi /(2 \alpha)] \bar{s}\left(l_{2 i}\right) 6 l_{2 i} \epsilon_{h}$ that appears from the definition (22). Now, suppose we get $\bar{s}\left(l_{0}\right)<\beta / \xi$ from the inequality (43). As explained, one can always find such $l_{0}$ for arbitrarily small $\beta$. We then find, using the subadditivity $\bar{s}\left(6 l_{2 i}\right)<\bar{s}\left(l_{2 i}\right)$ and the inequality (31), 
$\eta\left(2 l_{2 i}, l_{2 i}, 3 l_{2 i}\right)<6 \frac{\beta}{\alpha}\left(9 \gamma_{0}^{2}\right)^{i} l_{0}-2 \frac{\beta}{\alpha} \gamma_{0}^{i} \log \left\{\frac{\beta}{2 \alpha} \gamma_{0}^{i}\right\}$.

Apparently, $\sum_{i=0}^{\infty} \eta\left(2 l_{2 i}, l_{2 i}, 3 l_{2 i}\right)$ converges if $9 \gamma_{0}^{2}<1$, which is again the case for sufficiently small $\bar{s}\left(l_{0}\right)$. In the same manner, one can bound $\eta\left(2 l_{2 i+1}, l_{2 i+1}, 3 l_{2 i+1}\right)$, $\eta\left(l_{2 i}, l_{2 i}, l_{2 i}\right)$, and $\eta\left(l_{2 i+1}, l_{2 i+1}, l_{2 i+1}\right)$, and their summations over $i$, which completes the proof.

Note that in obtaining the inequality (50), we applied the inequality (31) with $\bar{s}\left(x^{n} l_{0}\right)<\gamma_{0}^{n} \bar{s}\left(l_{0}\right)$ instead of $\bar{s}\left(x^{n} l_{0}\right) \leq \prod_{i=0}^{n-1} \gamma\left(9,9^{i} l_{0}\right) \bar{s}\left(l_{0}\right)$, which is tighter. This was simply for brevity of the presentation. By replacing $\gamma_{0}^{i}$ in the inequality (50) with $\prod_{j=0}^{i-1} \gamma\left(9,9^{j} l_{0}\right)$, one obtains a much tighter bound. As the inequality (30) is nonlinear, this tighter bound should be obtained with the aid of numerical summation. In the next section, we present the details of the calculation.

\section{CALCULATION OF THE AREA-LAW BOUND}

As explained in the previous section, the proof is composed of two main steps. In the first step, we use Lemmas 4 and 5 to prove that there exists a certain $l_{0}$ such that $\bar{s}\left(l_{0}\right)$ is below a small threshold. In the second step, we use Lemmas $3,4,6$, and 7 to upper bound $S\left(4 \cdot 3^{n} l_{0}\right)$. The area-law bound is then $S(l) \leq 2 S\left(4 \cdot 3^{n} l_{0}\right)$ for arbitrary $l$ from the strong subadditivity of entropy.

\section{A. First step}

The following lemma incorporates Lemmas 4 and 5 .

Lemma 8. Fix $\alpha_{0} \in[2 / 3,1)$ and let $\ell_{n}=4^{n} \ell_{0}$ and $\bar{s}_{n}=\bar{s}\left(\ell_{n}\right)$ with

$$
\ell_{0}=\frac{2 \xi}{1-\alpha_{0}}\left(\log \frac{\xi}{1-\alpha_{0}}+3\right)
$$

Then, for any $Q_{c} \in[0,1]$,

$$
\begin{cases}\bar{s}_{n+1}<\bar{s}_{n}-Q_{c} \frac{\alpha_{0}}{4 \xi} & \text { if } \bar{s}_{n} \geq \frac{Q_{c}\left(1-Q_{c}\right) \alpha_{0}}{2\left(1-2 Q_{c}\right) \xi}>0 \\ \bar{s}_{n+1}<\frac{\bar{s}_{n}}{2\left(1-Q_{c}\right)} & \text { if } \bar{s}_{n} \leq \frac{Q_{c}\left(1-Q_{c}\right) \alpha_{0}}{2\left(1-2 Q_{c}\right) \xi}\end{cases}
$$

Proof.-Suppose $l_{B_{1}}: l_{A}: l_{B_{2}}=1: 2: 1$ and $l_{b}=\ell_{n}$; hence, $l_{C}=\ell_{n+1}$. Denote $Q\left(\alpha_{0}\right)$ in this setting by $Q_{n}$. Let $g=$ $8 \xi /\left(1-\alpha_{0}\right)$. Then, $\ell_{0}=(g / 4) \log g$. For this choice of $\ell_{0}, \epsilon\left(l_{C}, l_{b}, \alpha_{0}\right) \leq \epsilon\left(4 \ell_{0}, \ell_{0}, \alpha_{0}\right) \leq(7 g / 6)(\log g) 2^{-2 \log g}=$ $(7 / 6)(\log g) / g<\epsilon_{h}$ as $g \geq 24$. Lemmas 4 and 5 then imply

$$
\begin{aligned}
& \bar{s}_{n+1}<\frac{\bar{s}_{n}}{2\left(1-Q_{n}\right)}, \\
& \bar{s}_{n+1}<\bar{s}_{n}-Q_{n} \frac{\alpha_{0}}{4 \xi} .
\end{aligned}
$$

Note that for arbitrary $Q_{c}$, if $Q_{n} \leq Q_{c}, \quad \bar{s}_{n+1}<$ $\left\{\bar{s}_{n} /\left[2\left(1-Q_{c}\right)\right]\right\}$, and if $Q_{n} \geq Q_{c}, \bar{s}_{n+1}<\bar{s}_{n}-Q_{c}\left[\alpha_{0} /(4 \xi)\right]$. Thus,

$$
\bar{s}_{n+1}<\max \left\{\frac{\bar{s}_{n}}{2\left(1-Q_{c}\right)}, \bar{s}_{n}-Q_{c} \frac{\alpha_{0}}{4 \xi}\right\} .
$$

If $\bar{s}_{n} \geq\left\{\left[Q(1-Q) \alpha_{0}\right] /[2(1-2 Q) \xi]\right\}>0, \quad\left\{\bar{s}_{n} /[2(1-Q)]\right\} \leq$ $\bar{s}_{n}-Q\left[\alpha_{0} /(4 \xi)\right]$, and if $\bar{s}_{n} \leq\left\{\left[Q(1-Q) \alpha_{0}\right] /[2(1-2 Q) \xi]\right\}$, $\left[\bar{s}_{n} / 2(1-Q)\right] \geq \bar{s}_{n}-Q\left[\alpha_{0} /(4 \xi)\right]$. This observation leads to the lemma.

We are now in a position to find $l_{0}$ with a sufficiently small $\bar{s}\left(l_{0}\right)$. Our target is $\bar{s}\left(l_{0}\right)<\alpha_{0} / 27 \xi$.

Lemma 9. Take the definitions in Lemma 8. For any $\alpha_{0} \in[2 / 3,1)$, there exists $l_{0}$ and $n_{0}$ such that

$$
\begin{aligned}
l_{0} & \leq 4^{n_{0}} \ell_{0}, \\
n_{0} & \leq\left\lceil\frac{10 \xi}{\alpha_{0}}\left(1+\frac{\epsilon_{h}}{\ell_{0}}\right)\right\rceil+2, \\
\bar{s}\left(l_{0}\right) & <\frac{\alpha_{0}}{27 \xi},
\end{aligned}
$$

which implies

$$
S\left(l_{0}\right)<\frac{2 \alpha_{0}}{27\left(1-\alpha_{0}\right)}\left(\log \frac{\xi}{1-\alpha_{0}}+3\right) 4^{n_{0}} .
$$

Proof.-Let us use Lemma 8 with $Q_{c}=2 / 5$, for which

$$
\frac{Q_{c}\left(1-Q_{c}\right) \alpha_{0}}{2\left(1-2 Q_{c}\right) \xi}=\frac{3 \alpha_{0}}{5 \xi}
$$

Let $r=\left\lceil(10 \xi / \alpha)\left(1+\epsilon_{h} / \ell_{0}\right)\right\rceil$. If we assume $\bar{s}_{n} \geq 3 \alpha_{0} / 5 \xi$ for all integers $n \leq r-6$, then from the first inequality in Lemma 8,

$$
\frac{3 \alpha_{0}}{5 \xi} \leq \bar{s}_{r-6}<\bar{s}_{0}-(r-6) \frac{\alpha_{0}}{10 \xi}=\bar{s}_{0}-\frac{\alpha_{0}}{10 \xi} r+\frac{3 \alpha_{0}}{5 \xi}
$$

leads to a contradiction because this means

$$
\frac{\alpha_{0}}{10 \xi}\left\lceil\frac{10 \xi}{\alpha_{0}}\left(1+\frac{\epsilon_{h}}{\ell_{0}}\right)\right\rceil=\frac{\alpha_{0}}{10 \xi} r<\bar{s}_{0} \leq 1+\frac{\epsilon_{h}}{\ell_{0}} .
$$

We thus come to a conclusion that there exists an integer $n_{0}^{\prime} \leq r-6$ such that $\bar{s}_{n_{0}^{\prime}}<3 \alpha_{0} / 5 \xi$.

Once we arrive at this conclusion, we use the second inequality in Lemma 8 recursively with a proper choice of $Q_{c}$. In other words, as $\bar{s}_{n_{0}^{\prime}}<3 \alpha_{0} / 5 \xi$, we find $Q_{c}$ that satisfies $\left\{\left[Q_{c}\left(1-Q_{c}\right) \alpha_{0}\right] /\left[2\left(1-2 Q_{c}\right) \xi\right]\right\}=\left[\left(3 \alpha_{0}\right) /(5 \xi)\right]$, which is $Q_{c}=2 / 5$, so the second inequality in Lemma 8 is applicable. Then, the inequality indicates $\bar{s}_{n_{0}^{\prime}+1}<\alpha_{0} / 2 \xi$. Now, we find $Q_{c}$ that satisfies $\left\{\left[Q_{c}\left(1-Q_{c}\right) \alpha_{0}\right] /\left[2\left(1-2 Q_{c}\right) \xi\right]\right\}=$ $\left[\alpha_{0} /(2 \xi)\right]$, which is $Q_{c}=(3-\sqrt{5}) / 2 \simeq 0.38$, from which 
$\bar{s}_{n_{0}^{\prime}+2}<\alpha_{0} / 2(\sqrt{5}-1) \xi \simeq 0.40 \alpha_{0} / \xi$. We repeat this procedure as follows. For brevity, we present only numerical values up to the second significant digit: $\bar{s}_{n_{0}^{\prime}+2}<0.41 \alpha_{0} / \xi \rightarrow Q_{c}=$ $0.36 \rightarrow \bar{s}_{n_{0}^{\prime}+3}<0.32 \alpha_{0} / \xi \rightarrow Q_{c}=0.33 \rightarrow \bar{s}_{n_{0}^{\prime}+4}<0.24 \alpha_{0} /$ $\xi \rightarrow Q_{c}=0.28 \rightarrow \bar{s}_{n_{0}^{\prime}+5}<0.17 \alpha_{0} / \xi \rightarrow Q_{c}=0.23 \rightarrow \bar{s}_{n_{0}^{\prime}+6}<$ $0.11 \alpha_{0} / \xi \rightarrow Q_{c}=0.17 \rightarrow \bar{s}_{n_{0}^{\prime}+7}<0.064 \alpha_{0} / \xi \rightarrow Q_{c}=0.11 \rightarrow$ $\bar{s}_{n_{0}^{\prime}+8}<0.036 \alpha_{0} / \xi<\alpha_{0} / 27 \xi$. This implies the lemma.

\section{B. Second step}

The remaining task is to obtain the upper bound of the summation in Lemma 7. Let $l_{n} \equiv 3^{n} l_{0}$. As explained in the previous section, it is convenient to decompose the summation as $\sum_{i} \lambda_{i}=\sum_{m} \lambda_{2 m}+\sum_{m} \lambda_{2 m+1}$.

First, we need to obtain the upper bounds of $\left\{\bar{s}\left(l_{0}\right), \bar{s}\left(l_{2}\right), \bar{s}\left(l_{4}\right), \ldots\right\}$. For this, we choose $l_{B_{1}}: l_{A}: l_{B_{2}}=$ $1: 7: 1$ and $l_{b}=l_{2 m}$; hence, $l_{C}=l_{2 m+2}$. Let $Q_{2 m}$ denote $Q\left(\alpha_{0}\right)$ in this setting. Note that $l_{2 m}=9^{m} l_{0}$. We have

$$
\begin{aligned}
Q_{2 m} & \leq \frac{7 \xi}{\alpha_{0}} \bar{s}\left(7 l_{2 m}\right) \leq \frac{7 \xi}{\alpha_{0}} \bar{s}\left(l_{2 m}\right), \\
\bar{s}\left(l_{2 m+2}\right) & \leq \frac{1}{1-Q_{2 m}} \frac{2}{9} \bar{s}\left(l_{2 m}\right),
\end{aligned}
$$

where the first inequality uses Lemma 3 and the subadditivity (24), and the second inequality uses the inequality (29) originating from Lemma 4 . We thus obtain recursive inequalities with the initial condition $\bar{s}\left(l_{0}\right)<\left[\alpha_{0} /(27 \xi)\right]$. The first values are $Q_{0}<7 / 27$ and $\bar{s}\left(l_{2}\right)<\alpha_{0} / 90 \xi$. Then, for $m>0, \bar{s}\left(l_{2 m}\right)^{2} l_{2 m}$ decreases exponentially in $m$ as

$$
\frac{\bar{s}\left(l_{2 m+2}\right)^{2} l_{2 m+2}}{\bar{s}\left(l_{2 m}\right)^{2} l_{2 m}}<\frac{\bar{s}\left(l_{2}\right)^{2} l_{2}}{\bar{s}\left(l_{0}\right)^{2} l_{0}}=\frac{81}{100} .
$$

As stressed in the previous section, this exponential decay is an important point that results in the convergence of the summation in Lemma 7. We numerically obtain the upper bounds of $\left\{\bar{s}\left(l_{0}\right), \bar{s}\left(l_{2}\right), \bar{s}\left(l_{4}\right), \ldots\right\}$ for their use in the following calculation.

Consider the summation $\sum_{m=0}^{\infty} \lambda_{2 m}$. In order to use Lemma 6, we choose $\alpha=\alpha_{1}=\alpha_{0} / 2 \in[1 / 3,1 / 2)$. From Lemma 3 and the inequality (45) in Lemma 6 , we obtain

$$
\begin{aligned}
\eta\left(2 l_{2 m}, l_{2 m}, 3 l_{2 m}\right) \leq & 2 \frac{\xi}{2 \alpha_{1}} \bar{s}\left(l_{2 m}\right) S\left(6 l_{2 m}\right) \\
& -4 \frac{\xi}{2 \alpha_{1}} \bar{s}\left(l_{2 m}\right) \log \left\{\frac{\xi}{2 \alpha_{1}} \bar{s}\left(l_{2 m}\right)\right\} \\
& +2 \epsilon\left(l_{C}, l_{b}, 2 \alpha_{1}\right)+\epsilon\left(l_{B}+l_{b}, l_{b}, 2 \alpha_{1}\right) .
\end{aligned}
$$

As $\epsilon(\cdots)$ is much smaller than $\left(\xi / \alpha_{1}\right) \bar{s}\left(l_{2 m}\right) \epsilon_{h}$ and $\bar{s}\left(6 l_{2 m}\right) \leq \bar{s}\left(l_{2 m}\right)$ from the subadditivity (24),

$$
\begin{aligned}
& \eta\left(2 l_{2 m}, l_{2 m}, 3 l_{2 m}\right) \\
& \quad \leq \frac{12 \xi}{\alpha_{0}} \bar{s}\left(l_{2 m}\right)^{2} l_{2 m}-\frac{4 \xi}{\alpha_{0}} \bar{s}\left(l_{2 m}\right) \log \left\{\frac{\xi}{\alpha_{0}} \bar{s}\left(l_{2 m}\right)\right\} .
\end{aligned}
$$

Note that $\alpha_{1}$ was replaced by $\alpha_{0} / 2$. Similarly,

$$
\begin{aligned}
& \eta\left(l_{2 m}, l_{2 m}, l_{2 m}\right) \\
& \quad \leq \frac{12 \xi}{\alpha_{0}} \bar{s}\left(l_{2 m}\right)^{2} l_{2 m}-\frac{8 \xi}{\alpha_{0}} \bar{s}\left(l_{2 m}\right) \log \left\{\frac{2 \xi}{\alpha_{0}} \bar{s}\left(l_{2 m}\right)\right\} .
\end{aligned}
$$

Along with the upper bounds of $\left\{\bar{s}\left(l_{2 m}\right)\right\}$ obtained above, $\sum_{m=0}^{\infty} \lambda_{2 m}$ can be obtained numerically.

The summation $\sum_{m=0}^{\infty} \lambda_{2 m+1}$ is obtained similarly. Here, we frequently use the subadditivity, e.g., $\bar{s}\left(l_{2 m+1}\right) \leq \bar{s}\left(l_{2 m}\right)$, and $3 l_{2 m+1}=l_{2 m+2}$. We then obtain the following bounds, similar to the ones above:

$$
\begin{aligned}
& \eta\left(2 l_{2 m+1}, l_{2 m+1}, 3 l_{2 m+1}\right) \\
& \quad \leq \frac{4 \xi}{\alpha_{0}} \bar{s}\left(l_{2 m}\right) \bar{s}\left(l_{2 m+2}\right) l_{2 m+2}-\frac{4 \xi}{\alpha_{0}} \bar{s}\left(l_{2 m}\right) \log \left\{\frac{\xi}{\alpha_{0}} \bar{s}\left(l_{2 m}\right)\right\}, \\
& \eta\left(l_{2 m+1}, l_{2 m+1}, l_{2 m+1}\right) \\
& \quad \leq \frac{4 \xi}{\alpha_{0}} \bar{s}\left(l_{2 m}\right) \bar{s}\left(l_{2 m+2}\right) l_{2 m+2}-\frac{8 \xi}{\alpha_{0}} \bar{s}\left(l_{2 m}\right) \log \left\{\frac{2 \xi}{\alpha_{0}} \bar{s}\left(l_{2 m}\right)\right\} .
\end{aligned}
$$

From the set of upper bounds of $\left\{\bar{s}\left(l_{0}\right), \bar{s}\left(l_{2}\right), \bar{s}\left(l_{4}\right), \ldots\right\}$ and the inequalities (59)-(61), we obtain the following bound:

$$
\sum_{i=0}^{\infty} \lambda_{i}<0.1513 \frac{\alpha_{0}}{\xi} l_{0}+5.893 .
$$

By incorporating this and Lemma 9 into Lemma 7 and noting that $\eta\left(l_{n}, l_{n}, 2 l_{n}\right)$ is exponentially small in $n$, we arrive at the following lemma:

Lemma 10. Inherit the definitions in Lemma 9. For any $\alpha_{0} \in[2 / 3,1)$, there exists integer $N$ such that, for $n \geq N$,

$$
S\left(4 \cdot 3^{n} l_{0}\right)<\frac{\alpha_{0}}{2\left(1-\alpha_{0}\right)}\left(\log \frac{\xi}{1-\alpha_{0}}+3\right) 4^{n_{0}}+6 .
$$

\section{DISCUSSION}

The essential ingredient of the proof was defining the entropy per site $\bar{s}(l)$ and the cutoff index $q(\alpha)$ associated with the cutoff proportion $Q(\alpha)$. We obtained various relations of $\bar{s}(l)$ in terms of $Q(\alpha)$ for the partitioning in Fig. 1. An important observation is that as the length scale is increased as $\ell_{0} \rightarrow x \ell_{0} \rightarrow x^{2} \ell_{0} \rightarrow \cdots \rightarrow x^{n} \ell_{0}$ with some positive integer $x, \bar{s}\left(x^{n} \ell_{0}\right)$ asymptotically decays exponentially in $n$, and this is accompanied by the asymptotic exponential decay of mutual information $I(A: C)$ in $n$ when 
$\left\{l_{B_{1}}, l_{A}, l_{B_{2}}\right\}$ are all comparable to $x^{n} \ell_{0}$. This property motivated us to devise a renormalization-like construction in Fig. 2, revealing that the entropy of a large region is equivalent to the entropy of the fixed end regions up to the correction terms composed of the mutual information with different length scales. The correction terms are upper bounded by a constant thanks to the asymptotic decay of the mutual information.

Such an asymptotic behavior of $I(A: C)$ is, however, preceded by a transient stage in which $I(A: C)$ increases indefinitely. During the transient stage, the decrement of $\bar{s}\left(x^{n} \ell_{0}\right)$ is slower and $Q(\alpha)$ is unbounded [note that in Lemma 3, $Q(\alpha)$ can be bounded only when $\left.\bar{s}\left(l_{A}\right)<\alpha l_{b} / \xi l_{A}=\mathcal{O}[1 / \xi]\right]$. This transient stage persists until the length scale reaches a certain value $l_{0}$ that is exponentially large in $\xi$. Finding such $l_{0}$ was the first step of the previous section. This transient behavior is responsible for the area-law bound being exponentially large in $\xi$.

One can slightly modify the logic towards Lemma 6 in order to find the upper bound of $I\left(B_{1}: B_{2}\right)$ instead. In other words, we write the state as

$$
|\Psi\rangle=\sum_{i=1}^{M^{\prime}} \sqrt{p_{i}^{\prime}}\left|i^{\prime}\right\rangle^{B_{1}} \otimes\left|\phi_{i}{ }^{\prime}\right\rangle^{A B_{2} C}
$$

instead of Eq. (11) and define

$$
Q^{\prime}(\alpha)=\sum_{p_{i}^{\prime}<2^{-\alpha l_{A} / s}} p_{i}^{\prime}
$$

instead of Eq. (18). Then, we can obtain an upper bound similar to that in Lemma 6:

$$
\begin{aligned}
I\left(B_{1}: B_{2}\right) \leq & 2 Q^{\prime}(\alpha) S\left(B_{2}\right)-4 Q^{\prime}(\alpha) \log Q^{\prime}(\alpha) \\
& +2 \epsilon\left(l_{B_{2}}, l_{A}, \alpha\right)+\epsilon\left(l_{B}, l_{A}, 2 \alpha\right) .
\end{aligned}
$$

The behavior of $I(A: C)$ and $I\left(B_{1}: B_{2}\right)$-the initial increment, saturation, and the asymptotic decay-is a characteristic feature of the states with exponentially decaying correlations, although the initial growth of the mutual information may be absent. There would be various kinds of states with different behaviors of the mutual information. It is an interesting point that a state obeys an entanglement area law even when $I(A: C)$ asymptotically decays polynomially as $\mathcal{O}\left[n^{-k}\right]$ with $k>1$. It seems that there is room between the exponential and the polynomial decay, so the assumption (1) might be slightly mitigable, although we do not have a further result. This viewpoint suggests that the behavior of mutual information $I(A: C)$ in the IR limit is an important attribute governing the area-law scaling of entanglement entropies.

We note that the main assumption (1) can be generalized to some extent. Let $l_{R}=\min \left\{\left|R_{X}\right|,\left|R_{Y}\right|\right\}$ be the minimum size of $R_{X}$ and $R_{Y}$. Then, one can replace the assumption by

$$
|\langle X \otimes Y\rangle-\langle X\rangle\langle Y\rangle| \leq \operatorname{Poly}\left(l, l_{R}\right)\|X\|\|Y\| 2^{-l / \xi},
$$

where $\operatorname{Poly}\left(l, l_{R}\right)$ represents an arbitrary polynomial of $l$ and $l_{R}$. This change basically alters the form of $\epsilon\left(L, l_{b}, \alpha\right)$ in Eq. (21) and hence the unit length $\ell_{0}$ in Eq. (51). Otherwise, the structure of the proof is left unchanged because, throughout the proof, $l_{R}=\min \left\{l_{A}, l_{C}\right\}=l_{A}$ is always kept comparable to $l_{b}$; thus, the asymptotic behavior of $\epsilon\left(L, l_{b}, \alpha\right)$ is unchanged.

As a final remark, we note that the idea of taking a cutoff index $q(\alpha)$ is in line with the idea of approximating onedimensional many-body states with matrix product states [16]. In the language of the matrix product state, $q(\alpha)$ plays the role of a bond dimension, while $Q(\alpha)$ governs the accuracy of the approximation.

\section{ACKNOWLEDGMENTS}

This research was supported (in part) by the R\&D Convergence Program of NST (National Research Council of Science and Technology) of the Republic of Korea (Grant No. CAP-15-08-KRISS).

\section{APPENDIX: PROOF OF LEMMA 6}

(i) If $q(\alpha)=0$, and hence $Q(\alpha)=1$, the lemma follows from the triangle inequality $S(A)-S(C) \leq$ $S(A C)$.

(ii) Suppose $0<q(\alpha)<M$, and hence $0<Q(\alpha)<1$. We can split $\rho^{A C}$ into three parts:

$$
\begin{aligned}
\rho^{A C} & =\sum_{i, j=1}^{M} \sqrt{p_{i} p_{j}}|i\rangle^{A}\langle j| \otimes \phi_{i j}^{C} \\
& =\left[\sum_{i, j=1}^{q(\alpha)}+\sum_{i, j=q(\alpha)+1}^{M}+\sum_{\text {the rest }}\right] \sqrt{p_{i} p_{j}}|i\rangle^{A}\langle j| \otimes \phi_{i j}^{C} .
\end{aligned}
$$

Our aim here is to find the lower bound of $S\left(\rho^{A C}\right)$.

Let us first deal with the last sum. Suppose we add to the system a single qubit $a$ initialized in state $|0\rangle^{a}$ and apply a local unitary transformation on $a+A$ such that $|0\rangle^{a}|i\rangle^{A} \rightarrow|0\rangle^{a}|i\rangle^{A}$ for $i \leq q(\alpha)$ and $|0\rangle^{a}|i\rangle^{A} \rightarrow|1\rangle^{a}|i\rangle^{A}$ for $i>q(\alpha)$. The resulting state is

$$
\begin{aligned}
|\tilde{\Psi}\rangle= & \sqrt{1-Q(\alpha)}|0\rangle^{a}\left|\Psi_{1 q(\alpha)}\right\rangle \\
& +\sqrt{Q(\alpha)}|1\rangle^{a}\left|\Psi_{q(\alpha)+1, M}\right\rangle,
\end{aligned}
$$

where $\left|\Psi_{m n}\right\rangle \equiv\left(P_{m n} / \sqrt{Q_{m n}}\right)|\Psi\rangle$. Letting $\tilde{\rho}=$ $|\tilde{\Psi}\rangle\langle\tilde{\Psi}|$,

$\tilde{\rho}^{A C}=\left[\sum_{i, j=1}^{q(\alpha)}+\sum_{i, j=q(\alpha)+1}^{M}\right] \sqrt{p_{i} p_{j}}|i\rangle^{A}\langle j| \otimes \phi_{i j}^{C}$, 
which differs from Eq. (A1) by the last sum. Note that $|0\rangle^{a}\langle 0| \otimes \rho^{A C}$ and $\tilde{\rho}^{a A C}$ can be transformed into each other by a local unitary transformation on $a+A$, which implies $S\left(\rho^{A C}\right)=S\left(\tilde{\rho}^{a A C}\right)$. Thus, from the triangle inequality,

$$
S(A C)=S\left(\tilde{\rho}^{a A C}\right) \geq S\left(\tilde{\rho}^{A C}\right)-S\left(\tilde{\rho}^{a}\right) .
$$

Note that $S\left(\tilde{\rho}^{a}\right)=H(\{Q(\alpha), 1-Q(\alpha)\})$.
Let us now deal with the other two sums. We introduce another state

$$
\begin{aligned}
\tilde{\sigma}^{A C}= & \sum_{i=1}^{q(\alpha)} p_{i}|i\rangle^{A}\langle i| \otimes \phi_{i i}^{C} \\
& +\sum_{i, j=q(\alpha)+1}^{M} \sqrt{p_{i} p_{j}}|i\rangle^{A}\langle j| \otimes \phi_{i j}^{C},
\end{aligned}
$$

which differs from $\tilde{\rho}^{A C}$ by the off-diagonal terms in the first sum. We find

$$
\begin{aligned}
\mathcal{D}\left(\tilde{\rho}^{A C}, \tilde{\sigma}^{A C}\right) & =\frac{1}{2}\left\|\sum_{1 \leq i<j \leq q(\alpha)} \sqrt{p_{i} p_{j}}\left(|i\rangle^{A}\left\langle j\left|\otimes \phi_{i j}^{C}+\right| j\right\rangle^{A}\langle i| \otimes \phi_{j i}^{C}\right)\right\|_{1} \\
& \leq \frac{1}{2} \sum_{1 \leq i<j \leq q(\alpha)} \sqrt{p_{i} p_{j}} \||i\rangle^{A}\left\langle j\left|\otimes \phi_{i j}^{C}+\right| j\right\rangle^{A}\langle i| \otimes \phi_{j i}^{C} \|_{1} .
\end{aligned}
$$

Note

$$
\begin{aligned}
& \||i\rangle^{A}\left\langle j\left|\otimes \phi_{i j}^{C}+\right| j\right\rangle^{A}\langle i| \otimes \phi_{j i}^{C} \|_{1} \\
& \quad=\left\|X_{i j}^{A} \otimes\left(\phi_{i j}^{C}+\phi_{j i}^{C}\right)+Y_{i j}^{A} \otimes i\left(\phi_{i j}^{C}-\phi_{j i}^{C}\right)\right\|_{1} \\
& \quad \leq 2\left\{\left\|\phi_{i j}^{C}+\phi_{j i}^{C}\right\|_{1}+\left\|i\left(\phi_{i j}^{C}-\phi_{j i}^{C}\right)\right\|_{1}\right\},
\end{aligned}
$$

where $X_{i j}^{A}=|i\rangle^{A}\langle j|+| j\rangle^{A}\langle i|$ and $Y_{i j}^{A}=-i|i\rangle^{A}\langle j|+i| j\rangle^{A}\langle i|$ are the Pauli matrices. As the term inside the norm is Hermitian, having real eigenvalues, one can write, e.g., the first term as

$$
\left\|\phi_{i j}^{C}+\phi_{j i}^{C}\right\|_{1}=\operatorname{Tr}\left\{\left(\phi_{i j}^{C}+\phi_{j i}^{C}\right) \Lambda_{i j}^{C}\right\}
$$

for some matrix $\Lambda_{i j}^{C}$ that has \pm 1 as eigenvalues. Thus,

$$
\begin{aligned}
\sqrt{p_{i} p_{j}}\left\|\phi_{i j}^{C}+\phi_{j i}^{C}\right\|_{1} & =\left\langle X_{i j}^{A} \otimes \Lambda_{i j}^{C}\right\rangle \\
& \leq\left\langle X_{i j}^{A}\right\rangle\left\langle\Lambda_{i j}^{C}\right\rangle+\left\|X_{i j}^{A}\right\|\left\|\Lambda_{i j}^{C}\right\| 2^{-l_{b} / \xi} \\
& =2^{-l_{b} / \xi}
\end{aligned}
$$

where we use the Schmidt decomposition (11) in the first line, the assumption (1) in the second line, and $\left\langle X_{i j}^{A}\right\rangle=0$ and $\left\|X_{i j}^{A}\right\|=\left\|\Lambda_{i j}^{C}\right\|=1$ in the last line. The other norm is bounded in the same manner. We thus find
$\mathcal{D}\left(\tilde{\rho}^{A C}, \tilde{\sigma}^{A C}\right) \leq[$ of terms in Eq. (A5) $] 2 \cdot 2^{-l_{b} / \xi}$

$$
\leq 2 \frac{q(\alpha)\{q(\alpha)-1\}}{2} 2^{-l_{b} / \xi} \leq 2^{-(1-2 \alpha) l_{b} / \xi}
$$

where we use $q(\alpha) \leq 2^{\alpha l_{b} / \xi}$ (otherwise $\sum_{i=1}^{q(\alpha)} p_{i}>1$ ). Note that $\tilde{\sigma}^{A C}$ is purified by attaching a system with Hilbertspace dimension $q(\alpha)+1$ and the region $B$, while $\tilde{\rho}^{A C}$ is purified by attaching a qubit and the region $B$ (i.e., $|\tilde{\Psi}\rangle$ is the purification), which means $\tilde{\sigma}^{A C}$ has a larger Hilbertspace dimension. Furthermore, both the states share the same basis states. Consequently, the Fannes' inequality (19) can be applied with $d=\{q(\alpha)+1\} 2^{l_{B}}$. From $d \leq\left(2^{\alpha l_{b} / \xi}+1\right) 2^{l_{B}}<2^{l_{b}+l_{B}}$, it follows that

$$
\left|S\left(\tilde{\rho}^{A C}\right)-S\left(\tilde{\sigma}^{A C}\right)\right| \leq \epsilon\left(l_{B}+l_{b}, l_{b}, 2 \alpha\right) .
$$

We are now in a position to lower bound $S(A C)$. For convenience, let $\tilde{\sigma}_{m n}^{A C} \equiv\left(1 / Q_{m n}\right) P_{m n} \tilde{\sigma}^{A C} P_{m n}$. Then, Eq. (A4) can be written as

$$
\tilde{\sigma}^{A C}=\{1-Q(\alpha)\} \tilde{\sigma}_{1 q(\alpha)}^{A C}+Q(\alpha) \tilde{\sigma}_{q(\alpha)+1, M}^{A C} .
$$

Note

$$
\begin{aligned}
\{1-Q(\alpha)\} S\left(\tilde{\sigma}_{1 q(\alpha)}^{A C}\right) & =-\{1-Q(\alpha)\}\left\{\sum_{i=1}^{q(\alpha)} \frac{p_{i}}{1-Q(\alpha)} \log \frac{p_{i}}{1-Q(\alpha)}+\sum_{i=1}^{q(\alpha)} \frac{p_{i}}{1-Q(\alpha)} S\left(\phi_{i i}^{C}\right)\right\} \\
& =\sum_{i=1}^{q(\alpha)} p_{i}\left\{-\log p_{i}+S\left(\phi_{i i}^{C}\right)\right\}+\{1-Q(\alpha)\} \log \{1-Q(\alpha)\} \\
& \geq-\sum_{i=1}^{q(\alpha)} p_{i} \log p_{i}+\{1-Q(\alpha)\}\left\{S(C)-\epsilon\left(l_{C}, l_{b}, \alpha\right)\right\}+\{1-Q(\alpha)\} \log \{1-Q(\alpha)\},
\end{aligned}
$$


where the first line comes from a general equality $S\left(\sum_{i} p_{i}|i\rangle\langle i| \otimes \rho_{i}\right)=-\sum_{i} p_{i} \log p_{i}+\sum_{i} p_{i} S\left(\rho_{i}\right)$ satisfied when $\{|i\rangle\}$ is an orthonormal basis [24], and the third line is from Lemma 2. Note also

$$
\begin{aligned}
Q(\alpha) S\left(\tilde{\sigma}_{q(\alpha)+1, M}^{A C}\right) & \geq Q(\alpha) S\left(\tilde{\sigma}_{q(\alpha)+1, M}^{A}\right)-Q(\alpha) S\left(\tilde{\sigma}_{q(\alpha)+1, M}^{C}\right) \\
& \geq-Q(\alpha) \sum_{i=q(\alpha)+1}^{M} \frac{p_{i}}{Q(\alpha)} \log \frac{p_{i}}{Q(\alpha)}-\left[S\left(\tilde{\sigma}^{C}\right)-\{1-Q(\alpha)\} S\left(\tilde{\sigma}_{1 q(\alpha)}^{C}\right)\right] \\
& =-\sum_{i=q(\alpha)+1}^{M} p_{i} \log p_{i}+Q(\alpha) \log Q(\alpha)-Q(\alpha) S(C)-\{1-Q(\alpha)\} \epsilon\left(l_{C}, l_{b}, \alpha\right),
\end{aligned}
$$

where the first line comes from the triangle inequality of entropy, the second line from the concavity of entropy, and the third line from Lemma 2 and $S\left(\tilde{\sigma}^{C}\right)=S(C)$. From the inequalities (A3) and (A6), we have

$$
S(A C) \geq S\left(\tilde{\sigma}^{A C}\right)-S\left(\tilde{\rho}^{a}\right)-\epsilon\left(l_{B}+l_{b}, l_{b}, 2 \alpha\right) .
$$

Moreover, from Eqs. (A7)-(A9), and the concavity of entropy,

$$
\begin{aligned}
S\left(\tilde{\sigma}^{A C}\right) \geq & S(A)+S(C)-2 Q(\alpha) S(C) \\
& -H(\{Q(\alpha), 1-Q(\alpha)\})-2 \epsilon\left(l_{C}, l_{b}, \alpha\right) .
\end{aligned}
$$

Combining these two inequalities and noting $S\left(\tilde{\rho}^{a}\right)=$ $H(\{Q(\alpha), 1-Q(\alpha)\}) \leq-2 Q(\alpha) \log Q(\alpha)$ for $Q(\alpha) \leq 1 / 2$, the lemma follows.

(iii) If $q(\alpha)=M$, and hence $Q(\alpha)=0$, the extra qubit $a$ is not necessary anymore, and the inequality (A10) becomes $S(A C) \geq S\left(\tilde{\sigma}^{A C}\right)-\epsilon\left(l_{B}+l_{b}, l_{b}, 2 \alpha\right)$. Then, the lemma again follows from the inequality (A8) with $Q(\alpha)=0$.

[1] J. Eisert, M. Cramer, and M. B. Plenio, Colloquium: Area Laws for the Entanglement Entropy, Rev. Mod. Phys. 82, 277 (2010).

[2] M. B. Hastings and T. Koma, Spectral Gap and Exponential Decay of Correlations, Commun. Math. Phys. 265, 781 (2006).

[3] B. Nachtergaele and R. Sims, Lieb-Robinson Bounds and the Exponential Clustering Theorem, Commun. Math. Phys. 265, 119 (2006).

[4] D. Gosset and Y. Huang, Correlation Length versus Gap in Frustration-Free Systems, Phys. Rev. Lett. 116, 097202 (2016).

[5] M. B. Hastings, An Area Law for One-Dimensional Quantum Systems, J. Stat. Mech. (2007) P08024.

[6] D. Aharonov, I. Arad, U. Vazirani, and Z. Landau, The Detectability Lemma and Its Applications to Quantum Hamiltonian Complexity, New J. Phys. 13, 113043 (2011).

[7] I. Arad, Z. Landau, and U. Vazirani, Improved OneDimensional Area Law for Frustration-Free Systems, Phys. Rev. B 85, 195145 (2012).
[8] I. Arad, A. Kitaev, Z. Landau, and U. Vazirani, An Area Law and Sub-Exponential Algorithm for $1 D$ Systems, arXiv:1301.1162.

[9] N. de Beaudrap, M. Ohliger, T. J. Osborne, and J. Eisert, Solving Frustration-Free Spin Systems, Phys. Rev. Lett. 105, 060504 (2010).

[10] J. Cho, Sufficient Condition for Entanglement Area Laws in Thermodynamically Gapped Spin Systems, Phys. Rev. Lett. 113, 197204 (2014).

[11] M. B. Hastings, Entropy and Entanglement in Quantum Ground States, Phys. Rev. B 76, 035114 (2007).

[12] F. G. S. L. Brandão and M. Horodecki, An Area Law for Entanglement from Exponential Decay of Correlations, Nat. Phys. 9, 721 (2013).

[13] F. G. S. L. Brandão and M. Horodecki, Exponential Decay of Correlations Implies Area Law, Commun. Math. Phys. 333, 761 (2015).

[14] J. D. Bekenstein, Black Holes and Entropy, Phys. Rev. D 7, 2333 (1973).

[15] S. W. Hawking, Black Hole Explosions?, Nature (London) 248, 30 (1974).

[16] U. Schollwöck, The Density-Matrix Renormalization Group in the Age of Matrix Product States, Ann. Phys. (Amsterdam) 326, 96 (2011).

[17] R. Orús, A Practical Introduction to Tensor Networks: Matrix Product States and Projected Entangled Pair States, Ann. Phys. (Amsterdam) 349, 117 (2014).

[18] A. Kitaev and J. Preskill, Topological Entanglement Entropy, Phys. Rev. Lett. 96, 110404 (2006).

[19] M. Levin and X.-G. Wen, Detecting Topological Order in a Ground State Wave Function, Phys. Rev. Lett. 96, 110405 (2006).

[20] S. Ryu and T. Takayanagi, Holographic Derivation of Entanglement Entropy from the Anti-de Sitter Space/Conformal Field Theory Correspondence, Phys. Rev. Lett. 96, 181602 (2006).

[21] S. Gharibian, Y. Huang, Z. Landau, and S. W. Shin, Quantum Hamiltonian Complexity, arXiv:1401.3916.

[22] P. Hayden, D. Leung, P. W. Shor, and A. Winter, Randomizing Quantum States: Constructions and Applications, Commun. Math. Phys. 250, 371 (2004).

[23] M. Hastings, Random MERA States and the Tightness of the Brandao-Horodecki Entropy Bound, Quantum Inf. Comput. 16, 1228 (2016).

[24] M. Nielsen and I. Chuang, Quantum Computation and Quantum Information (Cambridge University Press, Cambridge, England, 2000). 